\title{
Traitement et typologie des variantes (para)synonymiques dans la lexicographie juridique française
}

\author{
Preite, Chiara \\ Università di Modena e Reggio Emilia \\ chiara.preite@unimore.it
}

Le langage juridique, très spécialisé, présente un haut degré de spécificité culturelle et une polysémie très riche où la synonymie $\mathrm{y}$ est remarquable. Cornu (2000: 178-182) propose une distinction entre synonymes - ou équivalents sémantiques totalement interchangeables - plutôt rares, et à peu près synonymes approximatifs ou parasynonymes ${ }^{1}-$ plus fréquents.

Rappelons cependant que, selon Lethuiller (1989 : 443), en langue de spécialité :

\begin{abstract}
il ne faut pas confondre synonymie et interchangeabilité en discours de formes linguistiques concurrentes. [...] des termes sont synonymes dès lors que leur fonction première, celle de désignation ou de pointeur de classe d'objets particuliers, est la même. Cela n'empêche pas qu'ils aient des attributs secondaires [...] différents et que, par conséquent, leur emploi soit plus adapté dans un type de discours que dans un autre. [...] Il y a synonymie lorsque plusieurs formes linguistiques ou termes servent à désigner ou pointer concurremment une même classe.
\end{abstract}

Ce parti pris, selon lequel il est possible de considérer deux variantes terminologiques comme des (para)synonymes indépendamment de leur interchangeabilité parfaite, nous suggère d'analyser la manière dont les lexicographes rendent compte de ces relations sémantiques dans cinq dictionnaires juridiques monolingues français sur papier, actuellement repérables sur le marché : Vocabulaire Juridique (VJ); Lexique des termes juridiques (LTJ); Dictionnaire du vocabulaire juridique (DVJ); Guide du langage juridique : vocabulaire - pièges et difficultés (GLJ); Vocabulaire du juriste débutant (VJD). Notre but est de proposer une typologie des variantes présentées et de vérifier leur traitement au niveau de micro- et medio-structure ${ }^{2}$.

\section{Caractéristiques des dictionnaires examinés}

En référence à la théorie des fonctions lexicographiques avancée Bergenholtz et Tarp $(1995 ; 2003)$ et développée par les lexicographes de l'Aahrus Center for Lexicography, nous pouvons affirmer que le LTJ et le DVJ se configurent comme des mini-encyclopédies juridiques en ce qu'ils ne visent que les fonctions knowledge-orientated; leurs informations touchent donc le savoir disciplinaire spécialisé. Le $\mathrm{VJ}$, tout en demeurant un dictionnaire knowledge-orientated, ne se limite pas à la présentation d'un savoir entendu comme discipline juridique, mais transmet également quelques notions relatives à la langue véhiculaire de cette discipline. Le GLJ accorde plus d'attention à l'usage de la langue et mélange des renseignements communication-orientated aux informations plus traditionnelles. Enfin, le VJD se détache du modèle classique ; il privilégie la réalisation des fonctions communication-orientated et propose des renseignements concernant un savoir(-faire) linguistique utile aux rédacteurs, traducteurs et étudiants. ${ }^{3}$

Sur la base des informations contenues dans les préfaces, présentations et avertissements des ouvrages consultés, nous observons, en premier lieu, que les dictionnaires s'adressent aux professionnels du droit ou de disciplines proches (donc aux experts), au grand public de non-experts pouvant s'intéresser à la terminologie juridique, et - nous ajoutons - aux traducteurs et interprètes qui sont généralement leurs utilisateurs principaux (Collet 2004). Dans tous les cas, l'usager principalement visé est l'étudiant en droit 
ou en langue juridique, donc semi-expert ou aspirant expert ; c'est pourquoi ces dictionnaires partagent un même souci pédagogique.

En deuxième lieu, soulignons que le but principal des dictionnaires juridiques est de fournir une description linguistique de la signification (et non seulement une description du concept, de l'objet ou de l'institution représentée par l'unité lexicale), apte à créer une représentation mentale partagée aussi bien par les experts que par les membres périphériques de la communauté de pratique (Wenger 1998) et de discours (Swales 1990 ; Beacco 1995 ; Beacco, Moirand 1995) visée.

Enfin, sans nous arrêter sur les différentes stratégies de représentation du sens, auxquelles les lexicographes peuvent avoir recours, nous nous limitons à noter que ces derniers optent, si possible, pour une définition par genre prochain et différence spécifique (cf. à ce propos Groffier, Reed 1990: 50-58), approfondie par le biais de descriptions encyclopédiques et explications de nature différente, ou pour l'emploi d'une variante (para)synonymique. Ainsi le VJD, qui se déclare " un dictionnaire de langue spécialisée » (Lerat 2007 : 3) et non de droit, réduit-il remarquablement les informations concernant la discipline au profit de la langue juridique. En effet, les renseignements que chaque article offre sur l'entrée ne concernent que les arguments qui la saturent dans son rôle de prédicat spécialisé ${ }^{4}$, et la paraphrase de sens est parfois remplacée par des (para)synonymes.

\section{2 (Para)synonymie et marques d'usage en lexicographie juridique}

Avant de passer à l'analyse des variantes (para)synonymiques contenues dans les dictionnaires juridiques, examinons ce que les lexicographes affirment à ce propos dans le paratexte de leurs ouvrages, ainsi que les stratégies de marquage appliquées dans les articles.

Cornu, qui a délibérément pris le parti, dans le VJ, de préciser les synonymes (VJ 2005 : X), explique les critères qu'il adopte pour présenter ses entrées. Parmi les renseignements fournis, il indique les renvois aux mots qui constituent le champ notionnel du défini, qui passent par les abréviations V., Comp., Syn., etc., lorsqu'il s'agit d'un renvoi «non intégré » (Wiegand 1992); et par l'astérisque * "en tête du mot auquel il est renvoyé, lorsque celui-ci figure dans le corps de la définition» (VJ 2005 : XIX), autrement dit en cas de «renvoi intégré ». En général, Cornu privilégie la marque Syn. pour signaler la présence de variantes (para)synonymiques, mais il emploie également les autres abréviations.

L'équipe dirigée par Guillien et Vincent dans la réalisation du LTJ ne mentionne nullement la prise en compte des variantes (para)synonymiques. Rappelons qu'il s'agit d'un groupe de juristes, moins préoccupés de la langue que de la discipline. Ce qui n'implique pas pour autant l'exclusion des (para)synonymes de ce dictionnaire ; ils y sont présents et introduits par des marques et expressions telles que : Synonyme de, Voir aussi, On dit également, etc.

Dans l'Avant-propos (2008: VIII) du DVJ, Cabrillac affirme que «Les définitions sont complétées, lorsque c'est possible et utile, par [...] un renvoi aux définitions des termes similaires ou connexes ». Ce renvoi est fait par le biais de plusieurs marques : Synonyme ou son abréviation Syn., Voir aussi (en fin d'article, en renvoi non intégré) et Voir (lorsque la définition est remplacée par un simple renvoi à un mot défini ailleurs).

Dans la Présentation (2007: 3) du VJD, Lerat fournit «un mode d'emploi » comprenant les quasisynonymes, qu'il exemplifie à travers le couple aliéner/céder. Cependant à la voix aliéner aucune mention n'est faite à céder et vice-versa. La plupart des renvois sont signalés tantôt par $c f$., ou tantôt par l'abréviation Syn.

L'originalité du GLJ de Bissardon consiste en la présence d'une annexe contenant une table des synonymes, qu'il présente comme suit (2005: 431):

Les synonymes permettent d'enrichir un acquis de vocabulaire et d'éliminer les répétitions disgracieuses. Ils constituent également un moyen de comprendre le sens des mots : pour connaître la signification d'un terme, il est souvent aussi efficace de 
jeter un œil sur la table des synonymes (ou d'antonymes) que de consulter un dictionnaire de langue.

Toutefois, l'auteur ne précise pas si les nombreux synonymes de cette table sont des termes interchangeables au regard du droit, ou s'il ne s'agit que de synonymies approximatives dont il n'explique pas les conditions d'emploi. De plus, bon nombre des termes apparaissant dans ces couples de variantes ne figurent pas dans la nomenclature du vocabulaire : le doute persiste donc. En revanche, en ce qui concerne le dictionnaire proprement dit, Bissardon emploie les marques Synonyme et Voir, ainsi que l'absence de relateur, $\varnothing$, comme dans l'exemple (1) où l'équivalent proposé ne reçoit pourtant pas de définition.

(1) Ester en justice. Agir en justice (GLJ)

Pour conclure ce rapide panorama sur les marques qui introduisent ou renvoient aux variantes (para)synonymiques, nous constatons l'absence de systématicité dans tous les dictionnaires. Les lexicographes emploient plusieurs marques: Syn(onyme de), Cf., Voir, Voir aussi, On dit également (LTJ), Est dit (LTJ), Est appelé (LTJ), Egalement nommé (VJ) Encore appelé (VJ), voire une absence de relateur qui se traduit par une simple juxtaposition, sans expliquer quels sont les critères de choix. L'absence de tout guide (précisant s'il s'agit de synonymes interchangeables, de variantes dans certains co(n)textes, ou s'il ne s'agit que d'analogies plus périphériques entre termes (notions voisines)) complexifie considérablement la compréhension de la part de l'usager non expert, de l'étudiant - qui en est pourtant le principal destinataire.

\section{Typologie des (para)synonymes en lexicographie juridique}

Nous allons donc essayer d'établir une classification des typologies (para)synonymiques repérées, à travers un répertoire limité mais représentatif d'exemples ${ }^{5}$.

\section{1 (Para)synonymie et renvois sans définition}

La plupart des renvois, et donc des variantes, se fondent sur la présentation d'un terme hyperonymique qui résume la catégorie conceptuelle de référence. Cependant il est possible de repérer des cas de correspondance biunivoque instaurée par le biais d'un renvoi direct. Ce dernier peut toucher des sigles et leurs syntagmes correspondants (cf. 3.3.), des emprunts et leurs francisations ou équivalents français (cf. 3.4.), mais aussi des exemples comme (2), où l'on cumule la relation synonymique et le renvoi :

(2) Juridiction ordinaire. Synonyme de juridiction de droit commun. Voir « juridiction de droit commun ». (GLJ)

Cette stratégie est employée également par le LTJ où l'on ne parle pas explicitement de synonymie mais on choisit $V$. et On dit également $(3 \mathrm{a}, 3 \mathrm{~b})$, et par le DVJ $(4 \mathrm{a}, 4 \mathrm{~b})$ qui choisit lui aussi deux renvois différents bien que réciproques, Synonyme de et Voir aussi : ce qui dénote un manque de cohérence dans la construction des renvois explicitant des relations sémantiques d'équivalence.

(3a) Heures de délégation. - Dr. trav. - V. Crédit d'heures. (LTJ)

(3b) Crédit d'heures. - Dr. trav. - Temps dont dispose un représentant du personnel ou un délégué syndical pour l'exercice de son mandat. On dit également « heures de délégation » [...]. (LTJ)

(4a) Accréditif. - Banc. Synonyme de lettre de crédit. (DVJ)

(4b) Lettre de crédit. - Banc. 1. Lettre par laquelle, dans l'opération de crédit documentaire, la banque émettrice informe le bénéficiaire de l'ouverture du crédit documentaire. 2. Document remis par un établissement de crédit à l'un de ses clients pour lui permettre d'obtenir un crédit auprès d'un banquier situé dans une autre place. Voir aussi : Accréditif. (DVJ) 
La correspondance biunivoque entre Accréditif et lettre de crédit est renforcée par le fait que ce dernier terme a deux acceptions et le renvoi reste valable pour les deux.

\section{2 (Para)synonymie pragmatique de type diachronique}

Les variantes diachroniques concernent la relation de correspondance biunivoque qui s'installe entre un terme venant du latin juridique et son équivalent français (5) :

(5) Placet. n.m. Acte par lequel est saisie une juridiction civile [...]. Syn. réquisition d'audience [...]. (VJD)

Le terme Réquisition d'audience n'apparaît pourtant pas dans la nomenclature du VJD, ni n'est signalé de renvoi à Placet à l'entrée Réquisition ou à Audience.

Un autre exemple concerne Possessio juris, Quasi-possession (6a, 6b) dont les renvois sont par contre réciproques :

(6a) Possessio juris. Expression latine signifiant «possession d'un droit » qui sert aujourd'hui à désigner toute *possession correspondant à un droit réel autre que la propriété. Syn. *quasi-possession. Comp. *possessio rei. (VJ)

(6b) Quasi-possession. Expression désignant la *possession correspondant à un droit réel autre que la propriété (servitude, usufruit). Syn. posessio juris. (VJ)

En ce qui concerne le GLJ, bon nombre de doublets latin/français ${ }^{6}$ trouvent place dans l'annexe consacrée au Synonymes. La simple forme de renvoi sans définition semble indiquer un rapport d'interchangeabilité biunivoque :

(7) Loi du for $\rightarrow$ Lex fori (GLJ)

La (para)synonymie diachronique connaît également d'autres formes. Observons l'exemple (8) :

(8a) Prêt. - Dr. civ. - [...] Le prêt de consommation est dit « $\rightarrow$ mutuum ». (V. ce $m o t)$ Le prêt à usage est appelé « $\rightarrow$ commodat». (V. ce mot) (LTJ)

(8b) Mutuum - Dr. civ. - Contrat en vertu duquel une personne, le prêteur, remet à une autre, l'emprunteur, pour qu'elle s'en serve, une chose fongible et consomptible, à charge pour elle d'en restituer une semblable. Ce contrat est aussi appelé prêt de consommation. Le «mutuum » est un contrat romain apparu à la fin de l'ancien droit. Le mot latin s'est conservé et habille aujourd'hui le prêt de consommation [...] $\rightarrow$ Prêt. (LTJ)

(8c) Commodat. - Dr. civ. - Prêt à l'usage ${ }^{7}$; il a pour objet une chose non consomptible qui doit être restituée par l'emprunteur en nature. Une proposition de loi sur la simplification du droit, votée en première lecture par l'Assemblée nationale et le Sénat (mars 2009) remplace le terme «commodat» par l'expression «prêt à usage ». $\rightarrow$ « Mutuum » ou prêt de consommation. (LTJ)

La notion hyperonymique de prêt contient deux hyponymes : le mutuum et le commodat. Dans les articles consacrés aux hyponymes - où l'on trouve les définitions respectives - nous découvrons que les deux ont été remplacés par les expressions plus modernes prêt à consommation et prêt à usage. Celles-ci ne jouissant pas pour autant d'articles indépendants, il est nécessaire de les chercher sous l'hyperonyme prêt. Le renvoi placé en fin d'article dans mutuum ( $8 \mathrm{~b}$ ) indique qu'il faut consulter prêt, alors que le renvoi à la fin de l'article commodat ne renvoie qu'à la notion voisine de mutuum ou à prêt de consommation. La reconstruction des champs lexical et notionnel s'avère dès lors complexe et non cohérente : les liens entre (para)synonymes diachroniques sont clairs, mais il n'en est pas de même pour les renvois entre cohyponymes et hyperonyme. 


\section{3 (Para)synonymie par siglaison}

Parfois, les dictionnaires offrent des sigles (opaques pour un public non expert ou semi-expert) en tant que variantes de termes complexes, composés d'un syntagme. Le rapport de correspondance sigle/syntagme résulte alors biunivoque $(9,10)$ :

(9a) EURL voir « entreprise unipersonnelle à responsabilité limitée ». (GLJ)

(9b) Entreprise unipersonnelle à responsabilité limitée (EURL) Société commerciale composée d'une seule personne [...]. (GLJ)

(10a) CMU. n.f. cf. couverture. (VJD)

(10b) Couverture. n.f. sing. Garantie contre les risques $>$ couverture sociale, couverture médicale universelle $(\boldsymbol{C M U}=$ accès gratuit aux soins médicaux $[\ldots])$. (VJD)

L'article consacré aux sigles se limite à renvoyer au syntagme correspondant. Le cas contraire peut se présenter, lorsque le syntagme ne fait pas partie de la nomenclature (11):

(11) CEDH. n.p.f.s. Cour européenne des Droits de l'homme (= «la Cour de Strasbourg », qui rend des arrêts à caractère purement déclaratoire mais suivis d'échos en droit interne). (VJD)

Il se peut également que le syntagme doive être cherché sous l'un de ses éléments (12) :

(12a) UE. V. Union Européenne. (VJ)

(12b) Union. [...] - Européenne (UE) : groupement des peuples et états d'Europe.

Des renvois comprenant quelques éléments additifs peuvent aussi être observés, comme dans le cas de CIF (13a), qui ne présente pourtant pas de définition. Celle-ci se trouve à CAF (13b), variante par francisation du sigle anglais correspondant (cf. 3.4.) :

(13a) CIF. Expression anglaise correspondant à l'expression française " ${ }^{*} \mathrm{CAF} »$, formée des initiales des trois mots : cost, insurance, freight. V. vente. (VJ)

(13b) CAF. Expression française formée des initiales des mots : coût, assurance, fret (comp. CIF) désignant une *vente de marchandises destinées à être transportées par mer, qui est faite moyennant un prix global comprenant le coût de la marchandise, le fret et l'assurance et comporte une livraison des marchandises à l'embarquement. (VJ)

Les deux expressions, qui sont de véritables synonymes forgés en co-rédaction et non des traductions, se renvoient de manière réciproque mais par des marques différentes (* et Comp.). Elles pourraient être considérées comme deux variantes diatopiques, dont l'option française est en tout cas favorisée dans la lexicographie juridique française.

\section{4 (Para)synonymie interlinguistique}

Selon Lethuiller (1989: 448) la synonymie de fond interlinguistique est abondante en langue de spécialité, et Lerat (2010) admet que «dans les terminologies orientées vers les termes ${ }^{8}$, on appelle traditionnellement équivalent une dénomination dénotant le même concept en langue étrangère ", bien qu'il considère que la dénomination de référence, ou terme normalisé, ne doit pas être mise sur le même plan que les autres, ou termes réels, parce que «le choix d'une dénomination de référence unique est nécessaire pour une gestion cohérente des concepts dénommés ».

Effectivement, les (para)synonymes interlinguistiques concernent certains emprunts (généralement anglicismes) ou greffes juridiques (Ewald 1995) dont les Commissions de Terminologie ont créé des francisations. C'est par exemple le cas de franchisage et franchising (14) :

(14) Franchisage. Subst. masc. - (Néol.). Terme substitué, par francisation, au mot angl. franchising [...]. Contrat - également nommé contrat de *franchise [...]. (VJ) 
Franchising ne fait pas l'objet d'une entrée dans les dictionnaires consultés : tous (sauf le GLJ qui dans la nomenclature n'adopte pas non plus le terme francisé ; cf. note 6) définissent le concept à l'entrée franchisage, et le DVJ n'indique même pas l'anglicisme correspondant. Ces choix paraissent refléter la politique linguistique prescriptive selon laquelle le terme français est à préférer. Considérons d'autres exemples, tirés du LTJ, où l'anglicisme et la francisation trouvent leur place : la tendance à la francisation avec simple mention de l'anglicisme correspondant est tantôt corroborée (15), et tantôt (16) le terme français ne renvoie pas au terme anglais bien que présent dans la nomenclature :

(15a) "Leasing". - Dr. com - V. Crédit-bail. (LTJ)

(15b) Crédit-bail. - Dr. com - Technique contractuelle moderne (d'origine américaine où elle porte le nom de leasing) [...]. (LTJ)

(16a) "Clearing house". - Dr. com. - V. Chambre de compensation. (LTJ)

(16b) Chambre de compensation. - Dr. com. - Réunion quotidienne ou pluriquotidienne des banquiers d'une même place afin de compenser leurs créances. (LTJ)

Le contraire peut parfois arriver aussi (17):

\section{(17a) Double renvoi (théorie du). Voir Foreign Court Theory (DVJ)}

(17b) Foreign Court Theory. - Dip. Théorie du renvoi en droit anglais dit « renvoi double » en ce qu'il autorise le juge du for à prendre en compte [...]. (DVJ)

La collocation de la définition à l'entrée étrangère nous laisse supposer que le terme d'origine est plus connu et répandu dans l'usage. Dans (17a) le syntagme français Double renvoi renvoie tout simplement à l'entrée consacrée à l'anglais Foreign Court Theory, alors que ce dernier ne renvoie pas à l'entrée correspondante en français. Dans (17b) le DVJ cite un "renvoi double», alors que l'entrée qui devrait correspondre est Double renvoi. Cette absence de correspondance complique le repérage de l'expression française par un usager non expert ou semi-expert, à l'encontre de la tendance générale à favoriser cette langue.

Comme nous l'avons mentionné pour franchisage et franchising (14), l'exemple (18) montre lui aussi une correspondance directe entre anglicisme et francisation, cette dernière étant pourvue de définition :

(18a) Engineering. V. Ingénierie (VJ)

(18b) Ingénierie. Subst. fém. - Néol. par francisation du terme angl. engineering, [...] Contrat complexe associant *entreprise, licence de brevet et communication de *savoir-faire, par lequel un entrepreneur s'engage vis-à-vis d'un donneur d'ordre à concevoir, installer et mettre en marche une unité de fabrication. V. know-how. (VJ)

Remarquons que (18b) montre aussi le renvoi à l'anglicisme know-how. Le couple know-how / savoirfaire se comporte de manière opposée : c'est le français savoir-faire (19a) qui renvoie à l'emprunt knowhow (19b) pour la définition.

(19a) Savoir-faire. V. know-how. (VJ)

(19b) know-how. Expression anglaise signifiant «savoir comment », à laquelle a été substituée, par *francisation, l'expression «*savoir-faire » [...], afin de désigner un ensemble de connaissances techniques [...]. (VJ)

Les variantes par francisation, ou tout simplement la co-existence d'un anglicisme et d'un terme correspondant français, consentent de remarquer que l'internationalisation croissante du domaine juridique consacre comme (para)synonymiques des expressions qui appartiennent à deux langues-cultures différentes. La manière dont les dictionnaires juridiques rendent compte de ces doublets, nous permet souvent de noter si les deux variantes sont également employées ou si l'une l'emporte sur l'autre. Par exemple, le français CAF (Côtt, Assurance, Fret) et son correspondant CIF (Cost, Insurance, Freight) semblent également courants, comme nous l'avons vu dans (13). 


\section{5 (Para)synonymie pragmatique de type diaphasique et diamésique}

Parmi les relations qui peuvent s'installer entre (para)synonymes juridiques, Cornu (2000 : 180-181) signale la présence dans le langage de doublets populaire-savant, dont seulement la valeur change, comme par exemple : Etablir (neutre) / Prouver (technique), Former appel (neutre) / Interjeter appel (imagé), Passer un bail (familier) / Conclure un bail (professionnel). Ces couples ne sont donc pas strictement interchangeables, car ils s'emploient dans des contextes différents bien que leurs sens soient très proches.

La recherche de ces doublets dans le VJ (en partie conçu par Cornu lui-même) montre que ces relations ne sont pas nécessairement explicitées, par exemple dans (20) le verbe Former ne constitue pas une entrée, ni l'expression n'est rapportée à l'entrée Appel.

(20) Interjeter [...] - appel. Faire appel ; accomplir l'acte par lequel l'appel est formé $[\ldots]$. (VJ)

Le linguiste juriste considère également une sorte de variation diamésique, en ce qu'il reconnaît que dans le style judiciaire oral du Palais certains termes sont employés comme synonymes, indépendamment de leurs différences $(21)^{9}$.

(21a) Demande en justice. La demande en justice est la voie ordinaire d'exercice du droit d'action. En ce sens, demande en justice est l'action en justice ou action. (VJ)

(21b) Action. [...] - Action en justice (toutes matières) [...] C / (par déviation) La *demande en justice, plus précisément la demande *principale, c'est-à-dire l'acte de procédure qui contient la prétention. (VJ)

Bien que le rapport entre demande et action en justice soit de type instrument/but, les deux termes sont souvent employés par les professionnels du domaine judiciaire comme synonymes interchangeables qui peuvent remplacer le terme prétention, qui devient un troisième élément (para)synonymique. En réalité, action et demande sont en rapport métonymique avec prétention (il s'agit de procédures contenant la prétention), mais ils finissent par remplacer ce terme dans le discours judiciaire.

\subsection{Acceptions et spécialisation de sens}

Dans certains cas, la (para)synonymie n'est ouvertement attribuée qu'à certaines acceptions ou spécialisation de sens :

(22a) Dommage. 1. Syn. (dans l'usage régnant) de *préjudice. *Atteinte subie par une personne dans son corps $[\ldots](\mathrm{VJ})$

(22b) Préjudice. 1. Syn. de *dommage dans l'usage régnant ; dommage subi par une personne dans son intégrité physique (VJ)

Cornu, de manière réciproque, indique que la synonymie (Syn.) entre dommage et préjudice est sanctionnée «dans l'usage régnant », explication aidant très peu un usager non ou semi-expert. Si nous consultons le LTJ nous retrouvons la même synonymie qui va de dommage à préjudice (22a bis), alors que dans la direction contraire elle n'est pas explicitée (22b bis).

(22a bis) Dommage. 1. Dans l'acception la plus courante, synonyme de préjudice (LTJ)

(22b bis) Préjudice. Dommage matériel [...], corporel [...] ou moral [...] subi par une personne par le fait d'un tiers. $\rightarrow$ Dommage (LTJ)

Il n'en demeure pas moins que, dans le LTJ aussi, cette synonymie ne fonctionne que « dans l'acception la plus courante ».

Il arrive donc qu'une acception d'un terme polysémique est employée comme variante (para)synonymique d'une acception d'un autre terme polysémique. C'est le cas, par exemple, de session et assises (23) : 
(23a) Session. 2. Période pendant laquelle *siège une juridiction non permanente, ainsi la *cour d'assise. Syn. *assises (sens 2). Comp. audience, séance, siège. (VJ)

(23b) Assises. 2 Par ext. la période pendant laquelle siège cette juridiction [...] ; les * sessions. (VJ)

Ce n'est que pour la deuxième acception du terme session que le dictionnaire signale comme Syn. la deuxième acception de assises. Dans (23b) nous retrouvons un renvoi implicite, car sans marque, à la forme du pluriel qui n’apparaît pas dans (23a).

\section{Conclusions}

La (para)synonymie, on le voit bien, est présente dans le langage juridique et les dictionnaires consacrés à ce domaine domaine en rendent compte de manière plus ou moins explicite et réglée.

D'abord nous avons relevé la variété de marques employées pour renvoyer aux (para)synonymes : Syn., Synonyme, Comp., Voir, Voir aussi, et $c f$. pour des segments non intégrés, un * en cas de segment intégré ; l'absence d'opérateur relationnel (Ø) lorsque l'expression remplace la définition. A cela s'ajoutent des expressions comme on dit également (LTJ), est dit (LTJ), est appelé (LTJ), également nommé (VJ), encore appelé (VJ), on l'appelle aussi (LTJ).

Cette pluralité de marques témoigne non seulement de l'intérêt porté à cette relation sémantique par tous les dictionnaires, mais aussi de l'absence de systématicité dans son traitement. Les rédacteurs des ouvrages consultés emploient ces marques et expressions sans expliciter quels en sont les critères de choix car, à l'exclusion de Lerat pour le VJD, ce ne sont pas des lexicographes experts ou des linguistes ; ils se montrent donc plus intéressés à la discipline juridique qu'à la cohérence micro- et mediostructurelle. Se mouvoir dans le labyrinthe de relations sémantiques et conceptuelles qui s'installent entre les variantes (para)synonymiques signalées (synonymes plus ou moins interchangeables, variantes contextuelles, analogies périphériques (notions voisines)) ne s'avère que plus complexe pour l'usager non expert et pour l'étudiant semi-expert. Ainsi, il devient ardu de comprendre si les variantes peuvent être interchangeables, et donc employées librement, ou si l'option se limite à certains contextes. Cette difficulté s'accentue lorsque la conceptualisation d'un terme n'est pas définie de manière pointue et rigoureuse, comme dans le cas des emprunts et des greffes juridiques.

Ensuite, nous avons essayé de dresser une typologie des (para)synonymes repérés dans les dictionnaires consultés selon le rapport qui s'installe entre variantes, en les subdivisant en 1) (para)synonymes unis par un renvoi sans définition ; 2) (para)synonymie pragmatique de type diachronique ; 3) (para)synonymie par siglaison ; 4) (para)synonymie interlinguistique, 5) (para)synonymie pragmatique de type diaphasique et diamésique ; 6) (para)synonymie qui concerne seulement des acceptions et spécialisation de sens. Comme l'ont montré les exemples cités et commentés, la présence d'exemples des typologies mentionnées dans un dictionnaire n'implique pas forcément une homogénéité de traitement.

Enfin, remarquons que les dictionnaires consultés ne présentent pas d'indications concernant l'«usage » au sens pragmatique de Desmet (2006: 242). En effet, le traitement lexicographique des termes, y compris celui qui naît de l'approche moins traditionnelle du lexique-grammaire que l'on trouve dans le VJD de Lerat, se limite à informer sur l'existence de variantes sans en préciser les différents contextes d'occurrence. Ces contextes représentent les réalisations discursives ou textuelles dans lesquelles ces termes sont acceptables: certaines variantes ne peuvent trouver d'emploi que dans des discours de vulgarisation, d'autres en revanche sont employées entre et par les professionnels dans des discours spécialisés.

\section{Références bibliographiques}

Beacco J.-C. (1995). A propos de la structuration des communautés discursives : beaux-arts et appréciatifs. In J.-C. Beacco, S. Moirand (éds.), Carnets du Cediscor, 3, Les enjeux des discours spécialisés. Paris : Presses de la Sorbonne Nouvelle, 135-157. 
Beacco J.-C., Moirand S. (1995). Autour des discours de transmission de connaissances. Langages, 117, 32-53.

Bergenholtz H., Tarp S. (1995). Manual of Specialized Lexicography, Amsterdam/Philadelphia : John Benjamins.

Bergenholtz H., Tarp S. (2003). Two Opposing Theories : On H.E. Wiegand's Recent Discovery of Lexicographic Functions. Hermes, Journal of Linguistics, 31, 171-196.

Bissardon S. (2005). Guide du langage juridique : vocabulaire - pièges et difficultés. Paris : Litec (II éd.).

Cabrillac R. et al. (2008). Dictionnaire du vocabulaire juridique. Paris : Litec (III éd.).

Collet T. (2004). Esquisse d'une nouvelle microstructure de dictionnaire spécialisé reflétant la variation en discours du terme syntagmatique. Meta, 49, 2, 247-263.

Cornu G. (2000). Linguistique juridique. Paris : Montchrestien (II éd.).

Cornu G. (2005). Vocabulaire juridique. Paris : Quadrige - PUF (VII éd.).

Desmet I. (2006). Variabilité et variation en terminologie et langues spécialisées : discours, textes et contextes. In Blampain D., Thoiron Ph., van Campenhoudt M. (éds.), Mots, termes et contextes. Paris : Ed. Archives contemporaines, 235-246.

Ewald W. (1995). The logic of legal transplants. American Journal of Comparative Law, 43, 489-510.

Groffier E., Reed D. (1990). La lexicographie juridique. Principes et méthodes. Cowanswille: Yvon Blais.

Guillien R., Vincent J. et al. (2010). Lexique des termes juridiques. Paris : Dalloz (XVII éd.).

Lerat P. (2002a). Vocabulaire juridique et schémas d'arguments juridiques. Meta, 47, 2, 155-162.

Lerat P. (2002b). Un niveau d'analyse privilégié pour les langues de spécialités européennes : le schéma d'énoncé. In Schena L., Soliman L.T. (éds.), Prospettive linguistiche della nuova Europa. Milano : Egea, 67-77.

Lerat P. (2005). Le vocabulaire juridique entre langue et texte. In Gémar J.-C., Kasirer N. (éds), Jurilinguistique. Montréal : Thémis, 59-70.

Lerat P. (2007). Vocabulaire du juriste débutant. Paris : Ellipses.

Lerat P. (2010). Variabilité et harmonisation terminologiques. In Atti Convegno Assiterm 2009, Terminologia, variazione $e$ interferenze linguistiche $e$ culturali, Publifarum, 12. http://publifarum.farum.it/ezine_articles.php?id=165

Lethuiller J. (1989). La synonymie en langue de spécialité, Meta, 34, 3, 443-449.

Preite C. (2012). Exemples de lexicographie juridique à orientation pédagogique en France : le Vocabulaire du juriste débutant et le Guide du langage juridique. In Vatvedt Fjeld R., Torjusen J.M. (éds.), Proceedings of the 15th Euralex International Congress. Sprakradet : Oslo, 570-577.

Preite C. (sous presse). La lexicographie spécialisée comme outil pédagogique : le cas du dictionnaire juridique en France. In Soliman L.T. (éd.), Termes en Discours, numéro spécial Travaux du Claix.

Swales J. (1990). Genre Analysis : English in Academic and Research Settings. Cambridge : Cambridge University Press.

Wenger E. (1998). Communities of Practice : Learning, Meaning and Identity. Cambridge : Cambridge University Press.

Wiegand H.E. (1992). Elements of a theory towards a so-called lexicographic definition. Lexicographica, 8, 175-285.

\footnotetext{
${ }^{1}$ Cette distinction est avancée aussi par Groffier et Reed (1990: 63), qui appellent analogies les à peu près et incluent les notions voisines dans cette catégorie.

${ }^{2}$ Il s'agit de la structure de renvois internes, ou cross-reference structure (Bergeholtz, Tarp 1995).

${ }^{3}$ Pour une étude approfondie sur les fonctions réalisées par ces dictionnaires, voir Preite (2012; sous presse).

${ }^{4}$ Voir à ce sujet Lerat (2002a, 2002b, 2005) et Preite (sous presse).
} 


\footnotetext{
${ }^{5}$ Pour former cette classification, nous avons adopté une démarche qualitative plutôt que quantitative, parce que les dictionnaires juridiques ne se basent pas sur des corpora et que les définitions ne sont pas construites par le biais de logiciels dédiés à la compilation lexicographique, mais ils sont le fruit du travail traditionnel et patient de leurs auteurs, ce qui entraîne - nous avons déjà eu l'occasion de le dire - un certain lot de non-systématicité.

${ }^{6}$ En revanche aucun doublet anglicisme/terme français ne se trouve dans cette annexe. Ces derniers sont placés dans une section faisant partie du Guide de rédaction, pièges et difficultés qui précède le dictionnaire proprement dit, dans lequel Bissardon présente les francisations devant remplacer les anglicismes (ex. Franchising, Franchisage, mais les deux ne font pas partie de la nomenclature).

${ }^{7}$ Ici, l'expression prêt à usage voit l'ajout de l'article défini.

${ }^{8}$ Suivant donc une approche sémasiologique, typique de la lexicographique.

${ }^{9}$ Par exemple, Action et demande en justice; Demander, requérir, réclamer; considérer, examiner; apprécier, estimer, etc. (Cornu $2000: 181$ ).
} 